\title{
Efeito do consumo de Kefir sobre parâmetros bioquímicos relacionados ao Diabetes Mellitus: uma revisão de literatura
}

\author{
Effect of Kefir intake on biochemical parameters related to Diabetes Mellitus: a literature review
}

Efecto del consumo de Kefir sobre parámetros bioquímicos relacionados con la Diabetes Mellitus: una revisión de la literatura

Camila Guazzelli Marques ${ }^{1}$, Renata Torres Alves ${ }^{1}$, Cintya Ji Young Park Lee ${ }^{1}$, Marcus Vinicius Lucio dos Santos Quaresma ${ }^{*}$

\section{RESUMO}

Objetivo: Revisar a literatura científica sobre a relação entre as intervenções realizadas com kefir e os parâmetros bioquímicos associados ao Diabetes Mellitus (DM). Métodos: Realizou-se uma busca bibliográfica nas bases de dados LILACS, PubMed e SciELO de junho a julho de 2018, considerando artigos em todos os idiomas, publicados nos últimos 10 anos. Os descritores utilizados para a pesquisa foram: "kefir", "diabetes mellitus", "glycemia", "probiotics", combinados com os conectores booleanos AND e OR. Resultados e Discussão: Foram selecionados 10 artigos na íntegra que atenderam os critérios estabelecidos, sendo 8 estudos realizados em animais e 2 em humanos. Os resultados evidenciados pelos estudos demonstraram efeitos positivos sobre os parâmetros bioquímicos associados a desordens glicêmicas apresentadas por animais ou humanos, como a glicemia de jejum e a hemoglobina glicada após diferentes períodos de intervenção. Os mecanismos subjacentes ainda são poucos esclarecidos, mas estudos sugerem que os efeitos positivos encontrados poderiam ser associados ao sinergismo entre as bactérias, leveduras e outros compostos bioativos encontrados no próprio kefir. Conclusão: O kefir parece apresentar potencial para ser utilizado no manejo das desordens glicêmicas em animais e humanos, entretanto, mais estudos clínicos, randomizados e controlados são necessários para confirmação.

Palavras-chave: Kefir, Diabetes Mellitus, Glicemia, Probióticos.

\begin{abstract}
Objective: Review the scientific literature on the relationship between interventions performed with kefir and the biochemical parameters associated with Diabetes Mellitus (DM). Methods: A bibliographic search was carried out in the LILACS, PubMed and SciELO databases from June to July 2018, considering articles in all languages, published in the last 10 years. The descriptors used for the research were: "kefir", "diabetes mellitus", "glycemia", "probiotics", combined with the Boolean connectors AND and OR. Results and Discussion: 10 articles were selected in full that met the established criteria, being 8 studies carried out in animals and 2 in humans. The results evidenced by the studies showed positive effects on the biochemical parameters associated with animal or human glycemic disorders, such as fasting glycemia and glycated hemoglobin after different intervention periods. The underlying mechanisms are still unclear, but studies suggest that the positive effects found could be associated with the synergism between bacteria, yeast and other bioactive compounds found in kefir. Conclusion: Kefir seems to present potential to be used in the
\end{abstract}

${ }^{1}$ Centro Universitário São Camilo, São Paulo - SP, Brasil. *E-mail: marcus.santos.nutri@gmail.com 
management of glycemic disorders in animals and humans, however, more clinical, randomized and controled studies are required for confirmation.

Keywords: Kefir, Diabetes Mellitus, Glycemia, Probiotics.

\section{RESUMEN}

Objetivo: Revisar la literatura científica sobre la relación entre las intervenciones realizadas con kefir y los parámetros bioquímicos asociados a la Diabetes Mellitus (DM). Métodos: Se realizó una búsqueda bibliográfica en las bases de datos LILACS, PubMed y SciELO de junio a julio de 2018, considerando artículos en todos los idiomas, publicados en los últimos 10 años. Los descriptores utilizados para la investigación fueron: "kefir", "diabetes mellitus", "glycemia", "probiotics", combinados con los conectores booleanos AND y OR. Resultados y Discusión: Se seleccionaron 10 artículos en su totalidad que atendieron los criterios establecidos, siendo 8 estudios realizados en animales y 2 en humanos. Los resultados evidenciados por los estudios demostraron efectos positivos sobre los parámetros bioquímicos asociados a desordenes glucémicos presentados por animales o humanos, como la glucemia de ayuno y la hemoglobina glucada después de diferentes períodos de intervención. Los mecanismos subyacentes todavía son pocos aclarados, pero los estudios sugieren que los efectos positivos encontrados podrían asociarse al sinergismo entre las bacterias, las levaduras y otros compuestos bioactivos encontrados en el propio kefir. Conclusión: El kefir parece presentar potencial para ser utilizado en el manejo de los desórdenes glucémicos en animales y humanos, sin embargo, más estudios clínicos aleatorizados y controlados son necesarios para confirmación.

Palabras-clave: Kefir, Diabetes Mellitus, Glicemia, Probióticos.

\section{INTRODUÇÃO}

O Diabetes Mellitus (DM) consiste em um distúrbio metabólico caracterizado pela hiperglicemia crônica, resultante da deficiência na produção ou na ação do hormônio pancreático insulina, ou ainda, em casos mais complexos, em ambos os mecanismos (SOCIEDADE BRASILEIRA DE DIABETES, 2017). O DM pode ser classificado de acordo com a sua etiologia, em tipo 1 (DM1), tipo 2 (DM2), gestacional (DMG) ou em tipos específicos devido a outras causas (AMERICAN DIABETES ASSOCIATION, 2018). Ainda, recentemente, alguns autores propuseram uma nova condição, o DM3, um distúrbio neuroendócrino caracterizado pela progressão da resistência central à insulina à doença de Alzheimer (MITTAL et al., 2016).

Independentemente do tipo ou da manifestação da doença, do seu grau de desenvolvimento ou de severidade, o DM é um importante e crescente problema de saúde pública no mundo (SOCIEDADE BRASILEIRA DE DIABETES, 2017). Globalmente, o número de pessoas com DM quadruplicou nas últimas três décadas, e dados a elencam nos dias atuais, como a nona principal causa de mortalidade (ZHENG et al., 2018) e com perspectivas futuras não promissoras. Em 2015 estimou-se que 415 milhões de adultos tinham diagnóstico de DM no mundo, sendo que mais de $90 \%$ dos casos correspondiam ao DM2 e, ainda mais alarmante, estimativas preveem um aumento para 642 milhões até 2040 (CHATTERJEE et al., 2017).

Nesse sentido, uma vez que o DM2 se mostra a forma mais comum da doença (PEREIRA et al., 2016), representando substancialmente a maior parte dos casos de DM no mundo, a compreensão dos fatores que desencadeiam a sua gênese se torna muito relevante aos profissionais do âmbito da saúde ou para pesquisadores da área. Segundo Zheng et al. (2018) os determinantes do DM2 consistem de uma matriz de fatores genéticos, epigenéticos e também de estilo de vida, que interatuam entre si e, ainda, agem dentro de um amplo ambiente físico-sociocultural.

Neste intrigante cenário, nos últimos anos pesquisadores buscam entender diariamente outros fatores que possam colaborar para a macro/micro compreensão do DM. Assim, recentemente, a microbiota intestinal (MI) 
tem sido sugerida como mais um fator contribuinte importante para o desenvolvimento de doenças crônicas, haja vista que distúrbios metabólicos, envolvendo o estresse oxidativo, inflamação sistêmica de baixo grau, resistência à insulina associam-se com alterações da composição e função da MI (EJTAHED et al., 2016; FORSLUND et al., 2015).

Ademais, considerando que a MI parece se relacionar diretamente com inúmeros distúrbios metabólicos, sobretudo àqueles relacionados ao metabolismo da glicose, envolvendo a resistência insulínica e DM, alguns autores propuseram que estratégias capazes de promover a integridade e funcionalidade da MI parecem ser relevantes na prevenção e no tratamento do DM de forma coadjuvante. Dentre as estratégias propostas, o manejo dietético vem sendo descrito como uma importante forma de modular a saúde da microbiota (DE FILIPPIS et al., 2016). Alguns autores já apontaram que padrões dietéticos ricos em alimentos com fibras, frutas e vegetais e de nutrientes anti-inflamatórios, características do padrão dietético mediterrâneo parecem influenciar diretamente e, modular de forma positiva a MI, aumentando a diversidade microbiana, em nível taxonômico ou genético (DE FILIPPIS et al., 2016). Além disso, outras estratégias alimentares como o aumento do consumo de fibras, prébióticos (frutooligossacarídeos e inulina) e probióticos têm sido propostas neste contexto (EJTAHED et al., 2016).

Em meio as estratégias dietéticas propostas, as intervenções com probióticos, atualmente, estão ganhando cada vez mais destaque no meio científico, principalmente pela sua capacidade em fornecer benefícios à saúde. O termo "probiótico" foi proposto pela primeira vez na década de 1960, sendo designado de forma geral para as espécies bacterianas consideradas benéficas para o trato gastrointestinal, mas somente em 2001 a Organização Mundial da Saúde (FAO/OMS, 2001) definiu oficialmente e especificamente os probióticos como sendo "microrganismos vivos que, quando administrados em quantidades adequadas, conferem um benefício à saúde do hospedeiro" (KHALESI et al., 2018).

Outra justificativa para esta notoriedade à suplementação probiótica se dá pelo rápido desenvolvimento tecnológico que possibilitou maior compreensão dos microrganismos que habitam e podem habitar o intestino do ser humano, sua funcionalidade e seus mecanismos de ação na saúde e na doença (SCHMIDT; RAES; BORK, 2018). Assim, os probióticos têm demonstrado ação na modulação da composição da microbiota intestinal, aumentando o número de bactéria benéficas e reduzindo o número de bactérias patogênicas, geralmente visualizado pelo aumento da razão bacterioidetes:firmicutes e, ainda, no aumento da diversidade de bactérias, em especial, uma bactéria que parece colaborar de forma determinante na saúde do hospedeiro, chamada de akkermansia muciniphila, a qual, está reduzida em indivíduos obesos e diabéticos (TORRESFUENTES et al., 2017).

Ademais, os probióticos atuam diretamente na integridade da barreira intestinal, reduzindo a permeabilidade do intestino e, por conseguinte, a translocação de bactérias ou fragmentos bacterianos que, sabidamente, estimulam em diferentes tecidos processos pró-inflamatórios e desordens no metabolismo (TORRES-FUENTES et al., 2017). Logo, os probióticos parecem agir de forma fundamental na regulação do metabolismo do hospedeiro e na prevenção e tratamento de distúrbios metabólicos envolvendo, sobretudo, a inflamação sistêmica de baixo grau (TORRES-FUENTES et al., 2017).

A recente meta-analise de He et al., (2017), a partir da inclusão de 10 ensaios clínicos randomizados de alta qualidade, demonstrou que os probióticos podem diminuir parâmetros negativos do perfil lipídico, pressão arterial e glicemia de jejum em pacientes com DM2. Outros estudos com diferentes cepas bacterianas também demonstraram efeitos positivos na obesidade em diferentes aspectos, tais como redução da inflamação do tecido adiposo, endotoxemia, adiposidade, níveis de leptina e consumo energético, sendo as espécies probióticas Bifidobacterium e Lactobacillus spp. as mais evidenciadas (TORRES-FUENTES et al., 2017).

Apesar dos benefícios descritos pela literatura, diversos clínicos questionam a viabilidade da prescrição probiótica, sobretudo por conta do alto valor que limita o acesso à diferentes populações. Ainda, Kadooka et al. (2013) demonstraram que quando a suplementação probiótica é cessada, os benefícios parecem reduzir, sugerindo a necessidade do consumo crônico. Considerando essa limitação prática, outros alimentos ricos 
em probióticos têm sido estudados, com intuito de viabilizar a prescrição, a fim de promover melhora da microbiota intestinal ao longo do tempo e, por conseguinte, benefícios à saúde do indivíduo.

Partindo deste pressuposto, há um recente interesse científico e popular por alimentos ou produtos fontes de probióticos, dentre eles, o kefir, que se originou nas montanhas do norte do Cáucaso (MAGALHÃES et al., 2011). O kefir diferencia-se de outros produtos lácteos basicamente pela presença do grão de kefir e da grande população de leveduras que apresenta (TAMANG et al., 2016). Em suma, trata-se de uma bebida de leite fermentada por uma matriz simbiótica de bactérias ácido-lácticas e leveduras que estão contidas dentro de um complexo composto por exopolissacarídeo e proteínas (WALSH et al., 2016). A bebida apresenta uma textura viscosa, de sabor azedo e acídico, baixos níveis de álcool e, em alguns casos, uma ligeira carbonatação, sendo tradicionalmente feita com leite de vaca, mas pode ser preparada com leite de outras fontes, como cabra, ovelha, búfalo ou extrato de soja (BOURRIE et al., 2016).

Estudos demonstraram que o consumo de kefir pode modular positivamente a microbiota intestinal, reduzir a inflamação sistêmica, o estresse oxidativo, mehorar a digestão da lactose e também condições clínicas como doenças intestinais inflamatórias, diabetes, dislipidemias e também diminuir a fadiga e melhorar o desempenho. (DESEENTHUM e JOHN, 2015; BOURRIE et al., 2016; HSU et al., 2018).

Nesse panorama, entende-se que a suplementação probiótica é limitada por fatores socioeconômicos e que outras estratégias, menos custosas, devem ser investigadas. Assim, essa revisão narrativa tem como objetivo principal revisar a literatura científica sobre a relação entre as intervenções realizadas com o kefir e os parâmetros bioquímicos associados ao DM.

\section{MÉTODOS}

Trata-se de uma revisão de literatura narrativa composta por publicações em periódicos internacionais. Realizou-se uma busca bibliográfica por meio de recursos eletrônicos nas bases de dados LILACS, PubMed e SciELO de junho a julho de 2018, considerando artigos em todos os idiomas e publicados no período de 2008 a julho de 2018.

Os descritores utilizados para a pesquisa foram: "kefir", "diabetes", "glycemia", "probiotics". Salienta-se que os descritores supracitados se encontram nos Descritores em Ciências da Saúde (DeCS). E os respectivos termos de pesquisa foram combinados com os conectores booleanos AND e OR.

Foram considerados critérios de inclusão, artigos realizados em modelo animal não humano (ratos) e em humanos, classificados como diabéticos, intervenções com kefir a curto, médio ou longo prazo e estudos com desfechos sobre parâmetros bioquímicos relacionados às desordens glicêmicas. Ademais, os estudos em humanos incluídos foram apenas os randomizados, duplo cego e placebo controlados.

\section{RESULTADOS}

Após a identificação dos artigos nas fontes de busca mencionadas, foram avaliados os títulos e os resumos, de modo a seleciona-los. Foram elencados 10 artigos na íntegra que atenderam os critérios de inclusão estabelecidos, bem como o objetivo do estudo, os quais foram incluídos na presente revisão.

Para adequada compreensão e extrapolação dos dados, os resultados foram apresentados separadamente em estudos realizados em modelos animais e em humanos por meio de dois diferentes quadros. No quadro 1 são apresentados 8 estudos realizados em animais e no quadro 2 são expostos os desfechos de 2 estudos em humanos. 
Quadro 1 - Estudos experimentais sobre Kefir e mudanças no metabolismo da glicose.

\begin{tabular}{|c|c|c|c|c|c|}
\hline Autor/Ano & Amostra & $\begin{array}{l}\text { Local de } \\
\text { obtenção do } \\
\text { kefir }\end{array}$ & Intervenção & $\begin{array}{l}\text { Duração } \\
\text { do } \\
\text { estudo }\end{array}$ & Principais Resultados \\
\hline $\begin{array}{l}\text { LEE et al., } \\
2011 .\end{array}$ & $\begin{array}{l}45 \text { Camundongos } \\
\text { diabéticos com } 5 \\
\text { semanas de idade. }\end{array}$ & $\begin{array}{l}\text { Não } \\
\text { Informado. }\end{array}$ & $\begin{array}{l}\text { Os animais foram divididos em três grupos ( } 15 \text { camundongos } \\
\text { cada): Grupo diabético tratados com solução salina; Grupo } \\
\text { diabético tratados com leite desnatado e Grupo diabético tratado } \\
\text { com kefir. }\end{array}$ & 45 dias & $\begin{array}{l}\cdot \downarrow \text { da glicemia no grupo kefir aos } 45 \text { dias em } \\
\text { comparação ao grupo tratado com solução salina e ao } \\
\text { grupo tratado com apenas leite desnatado }(p<0,05) \text {. }\end{array}$ \\
\hline $\begin{array}{l}\text { HADISAPUT } \\
\text { RO et al., } \\
2012 .\end{array}$ & $\begin{array}{l}84 \text { ratos Wistar machos, } \\
\text { com idade entre } 2,5 \text { a } 3 \\
\text { meses, pesando } 150- \\
250 \mathrm{~g} .\end{array}$ & $\begin{array}{l}\text { Casa de Kefir } \\
\text { Bening } \\
\text { Semarang. }\end{array}$ & $\begin{array}{l}\text { Condição diabética induzida por administração via } \\
\text { intraperitoneal com } 40 \mathrm{mg} / \mathrm{kg} \text { de peso de STZ. } \\
\text { Os animais foram divididos aleatoriamente em Grupo 1: animais } \\
\text { hiperglicêmicos que receberam tratamento à base de insulina } \\
\text { (0,76 Ul/200mg peso/dia); Grupo 2: animais hiperglicêmicos } \\
\text { tratados com kefir simples oral (3,6cc/200g peso/dia); Grupo 3: } \\
\text { apenas animais hiperglicêmicos (controle positivo); Grupo 4: } \\
\text { animais não hiperglicêmicos (controle negativo). }\end{array}$ & 30 dias & $\begin{array}{l}\cdot \downarrow \text { da glicemia no grupo tratado com insulina }(\Delta=- \\
162,29 \pm 76,74 \mathrm{mg} / \mathrm{dL}) ; \\
\cdot \downarrow \text { da glicemia no grupo tratado com } \operatorname{kefir}(\Delta=-111,00 \\
\pm 44,23 \mathrm{mg} / \mathrm{dL}) ;\end{array}$ \\
\hline $\begin{array}{l}\text { PUNARO et } \\
\text { al., } 2014 .\end{array}$ & $\begin{array}{l}42 \text { ratos Wistar machos, } \\
\text { com } 8 \text { semanas de } \\
\text { idade, pesando cerca de } \\
250 \mathrm{~g} \text {. } \\
\text { O DM foi definido neste } \\
\text { estudo como glicemia de } \\
\text { jejum } \geq 200 \mathrm{mg} / \mathrm{dL} \text {. }\end{array}$ & $\begin{array}{l}\text { Não } \\
\text { informado. }\end{array}$ & $\begin{array}{l}\text { Condição diabética induzida por administração intravenosa de } \\
45 \mathrm{mg} / \mathrm{kg} \text { de de STZ. } \\
\text { Os ratos foram alocados nos seguintes grupos: } C T L(n=9) \text { : grupo } \\
\text { controle; CTLK( } n=9) \text { : grupo controle tratado com a dose de } 1,8 \\
\mathrm{~mL} / \text { dia de kefir; } D M(n=12) \text { : grupo diabético e } \operatorname{DMK}(\mathrm{n}=12) \text { : grupo } \\
\text { diabético tratado com a dose de } 1,8 \mathrm{~mL} / \text { dia kefir). }\end{array}$ & $\begin{array}{c}8 \\
\text { semanas }\end{array}$ & $\begin{array}{l}\cdot \downarrow \text { do nível de glicose no sangue em jejum em ratos } \\
\text { DMK quando comparados aos ratos com DM } \\
\text { ( } p<0,001 \text { ) após tratamento; } \\
\text { - Durante o TOTG a glicemia no grupo DMK estava } \\
\text { diminuída após } 30,60 \text { e } 120 \text { min quando comparado } \\
\text { ao DM (embora isso tenha sido significativo apenas } \\
\text { após 30min }(p<0,05) \text {; }\end{array}$ \\
\hline $\begin{array}{l}\text { ALSAYADI } \\
\text { et al., } 2014 .\end{array}$ & $\begin{array}{l}24 \text { ratos Wistar adultos, } \\
\text { pesando } 200 \text { a } 250 \mathrm{~g} \text {. } \\
\text { Os animais com glicemia } \\
\text { de jejum }>200 \mathrm{mg} / \mathrm{dL} \\
\text { foram considerados } \\
\text { diabéticos e incluídos no } \\
\text { estudo. }\end{array}$ & $\begin{array}{l}\text { Os grãos de } \\
\text { kefir de água } \\
\text { foram obtidos } \\
\text { do laboratório } \\
\text { de YALACTA } \\
\text { (França) }\end{array}$ & $\begin{array}{l}\text { Condição diabética induzida STZ na dose de } 60 \mathrm{mg} / \mathrm{kg} \text { de massa } \\
\text { corporal. } \\
\text { Os ratos foram divididos em Grupo } 1 \text { (controle normal): dieta } \\
\text { padrão + água potável; Grupo 2: diabéticos que receberam dieta } \\
\text { padrão + água potável; Grupo 3 a 5: receberam dieta padrão e } \\
10 \%, 20 \% \text { e } 30 \% \text { de kefir inoculado na água potável, } \\
\text { respectivamente. }\end{array}$ & $\begin{array}{c}5 \\
\text { semanas }\end{array}$ & $\begin{array}{l}\cdot \downarrow \text { da glicemia ( } p \leq 0,05) \text { no final do experimento em } \\
\text { todos os grupos tratados com kefir inoculados na água } \\
\text { potável em comparação com ratos controle normais e } \\
\text { diabéticos; } \\
\text { - Não houve diferenças significativas entre os } 3 \\
\text { grupos tratados com kefir ( } p \leq 0,05) \text {; }\end{array}$ \\
\hline $\begin{array}{l}\text { NURLIYANI, } \\
\text { SADEWA, } \\
\text { SUNARTI, } \\
2015 .\end{array}$ & $\begin{array}{l}30 \text { ratos Wistar machos, } \\
\text { com } 8-12 \text { semanas de } \\
\text { idade. } \\
\text { Os animais com nível de } \\
\text { glicose em jejum }>126 \\
\mathrm{mg} / \mathrm{dL} \text {, foram }\end{array}$ & $\begin{array}{l}\text { Os grãos de } \\
\text { kefir foram } \\
\text { obtidos do } \\
\text { Centro de } \\
\text { Treinamento } \\
\text { de Pecuária }\end{array}$ & $\begin{array}{l}\text { Condição diabética induzida por } 60 \mathrm{mg} / \mathrm{kg} \text { de STZ e } 120 \mathrm{mg} / \mathrm{kg} \\
\text { de NA. Os ratos foram divididos em Grupo 1: controle negativo, } \\
\text { com apenas dieta padrão; Grupo 2: controle positivo, com ratos } \\
\text { diabéticos não tratados; Grupo } 3 \text { (kefir GM+BRE - } 1,0 \mathrm{ml} \text { ): ratos } \\
\text { diabéticos + kefir a } 1,0 \mathrm{ml} / 200 \mathrm{~g} \text { de massa corporal; Grupo } 4 \text { (kefir } \\
\text { GM+BRE - 2,0ml): ratos diabéticos + kefir a 2,0ml/200g de } \\
\text { massa corporal; Grupo } 5 \text { (kefir GM+BRE - } 4,0 \mathrm{ml} \text { ): ratos }\end{array}$ & 28 dias & $\begin{array}{l}\text { - } \uparrow \text { o número de células de Langerhans no grupo kefir } \\
\text { GM+BRE }(2,0 \mathrm{ml})(3,83 \pm 1,31) \text { comparado ao grupo } \\
\text { não tratado }(1,33 \pm 0,57)(p<0,05) \text {; } \\
\text { - } \uparrow \text { o número de células } \beta \text { positivas para insulina no } \\
\text { grupo kefir GM+BRE }(2,0 \mathrm{ml})(45,53 \pm 14,59) \\
\text { comparado ao grupo não tratado }(1,88 \pm 0,40)(p<0,05) ;\end{array}$ \\
\hline
\end{tabular}

REAS/EJCH | Vol.Sup.19 | e214 | DOI: https://doi.org/10.25248/reas.e214.2019 Página 5 de 12 


\begin{tabular}{|c|c|c|c|c|c|}
\hline & $\begin{array}{l}\text { considerados como } \\
\text { diabéticos. }\end{array}$ & $\begin{array}{l}\text { (Malang-East } \\
\text { Java, } \\
\text { Indonésia) }\end{array}$ & $\begin{array}{l}\text { diabéticos + kefir a } 4,0 \mathrm{ml} / 200 \mathrm{~g} \text { de massa corporal; Grupo } 5 \\
\text { (glibenclamida): ratos diabéticos + glibenclamida a } 50 \mathrm{mg} / \mathrm{kg} \text { de } \\
\text { massa corporal. }\end{array}$ & & $\begin{array}{l}-\uparrow \text { o número de Langerhans no grupo kefir GM+BRE } \\
(4,0 \mathrm{ml})(3,98 \pm 0,77) \text { comparado ao grupo não tratado } \\
(\mathrm{p}<0,05) ; \\
\text { - } \uparrow \text { o número de células } \beta \text { positivas para insulina no } \\
\text { grupo Kefir GM+BRE }(4,0 \mathrm{ml})(45,69 \pm 17,46) \\
\text { comparado ao grupo não tratado }(p<0,05) ;\end{array}$ \\
\hline $\begin{array}{l}\text { MUNTAFIAH } \\
\text { et al., } 2015 .\end{array}$ & $\begin{array}{l}25 \text { ratos Wistar machos, } \\
\text { com } 12 \text { semanas, } \\
\text { pesando } 200 \text { a } 300 \mathrm{~g} . \\
\text { Foram considerados } \\
\text { hiperglicêmicos, com } \\
\text { nível de glicose em jejum } \\
>126 \mathrm{mg} / \mathrm{dL} .\end{array}$ & $\begin{array}{l}\text { Não } \\
\text { informado. }\end{array}$ & $\begin{array}{l}\text { Condição diabética induzida por } 60 \mathrm{mg} / \mathrm{kg} \text { de massa corporal de } \\
\text { STZ e } 120 \mathrm{mg} / \mathrm{kg} \text { de massa corporal de NA. Os animais foram } \\
\text { divididos em } 5 \text { grupos: grupo controle saudável, grupo controle } \\
\text { diabético, grupo tratado com } 100 \% \text { leite de cabra kefir, grupo } \\
100 \% \text { extrato de soja kefir e grupo combinação de } 50 \% \text { kefir de } \\
\text { leite de cabra e } 50 \% \text { kefir extrato de soja. }\end{array}$ & $\begin{array}{c}4 \\
\text { semanas }\end{array}$ & $\begin{array}{l}\cdot \downarrow \text { da glicemia de jejum no grupo tratado com } 100 \% \\
\text { leite de cabra kefir após período de intervenção } \\
\text { ( } p=0,073) \\
\cdot \downarrow \text { da glicemia de jejum no grupo tratado com grupo } \\
100 \% \text { extrato de soja kefir após período de } \\
\text { intervenção ( } p=0,646) \\
\cdot \downarrow \text { da glicemia de jejum no grupo tratado com a } \\
\text { combinação de } 50 \% \text { kefir de leite de cabra e } 50 \% \text { kefir } \\
\text { extrato de soja }(p<0,05) \text {. }\end{array}$ \\
\hline $\begin{array}{c}\text { NURLIYANI, } \\
\text { HARMAYAN } \\
\text { I, SUNARTI, } \\
2015 .\end{array}$ & $\begin{array}{l}30 \text { ratos Wistar machos, } \\
\text { com idade entre } 8 \text { e } 12 \\
\text { semanas. Foram } \\
\text { considerados diabéticos, } \\
\text { se } 7 \text { dias após a indução, } \\
\text { a glicemia de jejum era } \\
\text { maior que } 126 \mathrm{mg} / \mathrm{dL} \text {. }\end{array}$ & Não informado & $\begin{array}{l}\text { Condição diabética induzida por } 60 \mathrm{mg} / \mathrm{kg} \text { de massa corporal de } \\
\text { STZ e } 120 \mathrm{mg} / \mathrm{kg} \text { de massa corporal de NA. } \\
\text { Os animais foram distribuídos aleatoriamente em } 5 \text { grupos ( } \mathrm{n}=6 \\
\text { por grupo). Grupo 1: controle negativo (ratos normais); Grupo 2: } \\
\text { controle positivo (ratos diabéticos); Grupo 3: ratos diabéticos } \\
\text { tratados com leite de cabra kefir; Grupo 4: ratos diabéticos } \\
\text { tratados com kefir de leite de cabra e extrato de soja; Grupo 5: } \\
\text { ratos diabéticos alimentados com extrato de soja kefir. }\end{array}$ & 35 dias & $\begin{array}{l}\cdot \downarrow \text { da glicemia nos ratos diabéticos + leite de cabra } \\
\text { kefir }(\Delta=-92,36 \pm 116,05 \mathrm{mg} / \mathrm{dL}) ; \\
\cdot \downarrow \text { da glicemia nos ratos diabéticos + leite de cabra e } \\
\text { extrato de soja kefir }(\Delta=-225,95 \pm 36,71 \mathrm{mg} / \mathrm{dL}) ; \\
\cdot \downarrow \text { glicemia nos ratos diabéticos }+ \text { extrato de soja } \\
\text { kefir }(\Delta=-133,85 \pm 172,37 \mathrm{mg} / \mathrm{dL}) ; \\
\cdot>\text { número médio de ilhotas de Langerhans dos ratos } \\
\text { diabéticos tratados ratos diabéticos + leite de cabra e } \\
\text { extrato de soja kefir quando comparado aos diabéticos } \\
\text { não tratados }(p<0,05) ;\end{array}$ \\
\hline $\begin{array}{l}\text { SUNARTI, } \\
\text { NURLIYANI, } \\
\text { TYAS, } 2015 .\end{array}$ & $\begin{array}{l}25 \text { ratos Wistar machos, } \\
(200-300 \mathrm{~g} \text { com } 3 \text { meses } \\
\text { de idade. Os ratos foram } \\
\text { considerados como } \\
\text { diabéticos se tivessem } \\
\text { níveis de glicose } \\
\text { plasmática }>280 \mathrm{mg} / \mathrm{dL} \text {. }\end{array}$ & Não informado & $\begin{array}{l}\text { Condição diabética induzida 120mg/kg de massa corporal de NA } \\
\text { e } 60 \text { mg/kg de massa corporal de STZ. } \\
\text { Os animais foram divididos aleatoriamente em cinco grupos: } \\
\text { Grupo 1: ratos normais; Grupo 2: ratos diabéticos; Grupo 3: } \\
\text { ratos diabéticos com kefir de leite de cabra; Grupo } 4 \text { ratos } \\
\text { diabéticos com consumo de kefir de extrato de soja; Grupo 5: } \\
\text { ratos diabéticos com uma combinação de kefir de leite de cabra } \\
\text { e soja. }\end{array}$ & $\begin{array}{c}4 \\
\text { semanas }\end{array}$ & $\begin{array}{l}\cdot \downarrow \text { da glicemia nos ratos diabéticos tratados com kefir } \\
\text { de leite de cabra ( } p=0,021) \text {; } \\
\text { - } \downarrow \text { da glicemia em ratos diabéticos com combinação } \\
\text { de leite de cabra e extrato de soja kefir ( } p<0,001 \text { ); } \\
\text { - } \downarrow \text { da glicemia nos animais diabéticos tratados com } \\
\text { kefir de extrato de soja ( } p=0,0157) ;\end{array}$ \\
\hline
\end{tabular}

2; $\mathrm{HbA} 1 \mathrm{c}=$ hemoglobina glicada; $\mathrm{p}<0,05=$ resultado significativo; $p<0,001=$ resultado significativo]
.

Fonte: Dados da pesquisa, 2018.

REAS/EJCH | Vol.Sup.19 | e214 | DOI: https://doi.org/10.25248/reas.e214.2019 Página 6 de 12 
Quadro 2 - Estudos clínicos que verificaram o efeito do Kefir nas desordens glicêmicas.

\begin{tabular}{|c|c|c|c|c|c|}
\hline Autor/Ano & Amostra & $\begin{array}{l}\text { Local de } \\
\text { obtenção } \\
\text { do kefir }\end{array}$ & Intervenção & $\begin{array}{c}\text { Duração do } \\
\text { estudo }\end{array}$ & Principais resultados nos parâmetros glicêmicos \\
\hline $\begin{array}{c}\text { JUDIONO et al., } \\
2014 .\end{array}$ & $\begin{array}{l}108 \text { indivíduos DM2, } \\
\text { sendo o último nível de } \\
\text { glicose no sangue em } \\
\text { cerca de <200 mg/dL, } \\
\text { equivalente a HbA1c } \\
6-8 \% \text {. }\end{array}$ & $\begin{array}{l}\text { Casa de } \\
\text { kefir Bening } \\
\text { Semarang. }\end{array}$ & $\begin{array}{l}\text { Os pacientes foram divididos } \\
\text { aleatoriamente em } 3 \text { grupos: grupo } 1 \\
\text { (DM2 com HbA1c < } 7,0 \% \text {, alimentado } \\
\text { com dieta padrão e suplementado com } \\
200 \mathrm{ml} / \text { dia de kefir), grupo } 2 \text { (DM2 com } \\
\mathrm{HbA} 1 \mathrm{c}>7,0 \% \text {, alimentado com dieta } \\
\text { padrão e suplementado com } 200 \\
\text { ml/dia de kefir) e grupo } 3 \text { (DM2 com } \\
\text { tratado com uma dieta padrão e sem } \\
\text { kefir, sendo grupo controle). }\end{array}$ & 30 dias & $\begin{array}{l}\cdot \downarrow \text { Os níveis de glicose no sangue em jejum e a glicose pós } \\
\text { pradial nos três grupos após intervenção, mas os grupos } \\
\text { tratados com kefir demostraram diminuições mais expressivas } \\
\text { em relação ao grupo controle; } \\
\text { • } \uparrow \text { nos valores de peptídeo C nos grupos tratados com kefir } \\
\text { após interveção comparado ao grupo não tratado }(p=0,014) \text {; } \\
\text { • } \downarrow \text { da HbA1c nos grupos tratados com kefir em comparação ao } \\
\text { grupo controle após o período de intervenção ( } p=0,001) \text {; }\end{array}$ \\
\hline $\begin{array}{l}\text { OSTADRAHIMI } \\
\text { et al., } 2015 .\end{array}$ & $\begin{array}{l}60 \text { pacientes DM2 com } \\
\text { idade entre } 35 \text { a } 65 \\
\text { anos. Foram incluídos } \\
\text { neste estudo apenas } \\
\text { indivíduos diabéticos } \\
\text { com glicemia em jejum } \\
\geq 125 \mathrm{mg} / \mathrm{dl} \text {, idade } \\
\text { entre } 35 \text { e } 65 \text { anos, } \\
\text { sem insulinoterapia e } \\
\text { com duração da } \\
\text { doença menor que } 20 \\
\text { anos. }\end{array}$ & $\begin{array}{l}\text { Goldam } \\
\text { Dairy } \\
\text { Company, Ir }\end{array}$ & $\begin{array}{l}\text { Grupo intervenção: tratado com } 600 \mathrm{ml} \\
\text { de leite fermentado (kefir) contendo } \\
\text { probióticos duas vezes ao dia (almoço } \\
\text { e jantar); } \\
\text { Grupo placebo: tratado com } 600 \mathrm{ml} \text { de } \\
\text { leite fermentado convencional (massa) } \\
\text { também duas vezes ao dia (almoço e } \\
\text { jantar). }\end{array}$ & 8 semanas & $\begin{array}{l}-\downarrow \downarrow \text { significativa da glicemia em jejum no grupo kefir após } \\
\text { tratamento; } \\
\text { - } \downarrow \text { significativa da HbA1c no grupo kefir após tratamento; }\end{array}$ \\
\hline
\end{tabular}

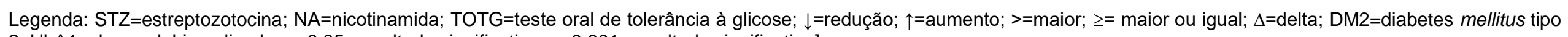
2; HbA1c=hemoglobina glicada; $p<0,05=$ resultado significativo; $p<0,001=$ resultado significativo]

Fonte: Dados da pesquisa, 2018. 


\section{DISCUSSÃO}

Até o presente momento, à luz de nosso conhecimento, há poucos estudos intervencionais publicados em humanos, sendo a maioria das pesquisas conduzidas em modelos animais. Neste cenário, Hadisaputro et al. (2012) justificaram a escolha pelos modelos animais, não só pela facilidade metodológica, mas por ainda ser necessário elucidar os mecanismos de ação do kefir, antes de utiliza-lo como estratégia de intervenção no ser humano. Todavia, é interessante verificar que o consumo de kefir mostra-se extremamente amplo pela população, muito embora, o nível de evidência científica sobre o tema seja baixo.

De tal modo, Lee et al. (2011) investigaram o efeito da administração oral de kefir sobre os níveis de glicose sanguínea em camundongos diabéticos. O kefir foi inoculado no leite desnatado e administrado via oral a 10 $\mathrm{mL} / \mathrm{kg}$ de massa corporal, duas vezes ao dia por um período de 45 dias, período suficiente para os autores verificaram que a glicemia do grupo tratado com kefir diminuiu significativamente $(p<0,05)$ em comparação aos outros dois grupos. Logo, neste estudo o kefir pareceu atuar beneficamente na regulação da glicemia de animais diabéticos após 45 dias.

Posteriormente, Hadisaputro et al. (2012) também verificaram o efeito do kefir obtido a partir da fermentação por grãos de kefir inoculados no leite desnatado por 24 horas sobre a glicemia de animais induzidos à hiperglicemia durante 30 dias. Em suma, os resultados evidenciados demonstraram redução na glicemia no grupo tratado com insulina (delta $(\Delta)=-162,29 \pm 76,74 \mathrm{mg} / \mathrm{dL}$ ) e também no grupo tratado com kefir $(\Delta=-111,00 \pm 44,23 \mathrm{mg} / \mathrm{dL}$ ), enquanto que no grupo de animais hiperglicêmicos sem tratamento (controle positivo) a glicemia aumentou ao final da interveção $(\Delta=+41,28 \pm 53,19 \mathrm{mg} / \mathrm{dL})$. A regulação glicêmica após administração de insulina já era esperada nestes animais, mas interessantemente o kefir também apresentou potencial para desencadear tal efeito clínico.

A hipótese mecanística para explicar os resultados positivos sugeridos pelo kefir baseia-se principalmente pelos seus exopolissacarídeos bioativos (EPS) que parecem deflagrar um processo em cascata resultando em maior liberação de insulina pelas células $\beta$ pancreáticas, influenciando o aumento na captação de glicose pelas células e tecidos periféricos. Este mecanismo também é sugerido e ainda mais detalhado no estudo apresentado posteriormente por JUDIONO et al., 2014 realizado em humanos. Ademais, o kefir além de reduzir a glicemia nestes animais, reduziu as concentrações de citocinas pró-inflamatórias, (IL-1 e TNF- $\alpha$ ). Inclusive, a redução das citocinas também justifica a razão pela qual a glicemia melhorou. Acredita-se que 0 aumento do processo inflamatório possa colaborar para piora da secreção e da sensibilidade à insulina. Logo, os autores concluem que o kefir pode prevenir a glicotoxicidade e a lipotoxicidade, além de reduzir a ocorrência de hiperglicemia, e que tais desfechos estariam associados ao sinergismo entre as bactérias, leveduras e outros compostos bioativos encontrados no kefir.

Haja vista que poucos estudos realizados com kefir apresentaram os ingredientes e doses utilizadas no tratamento, Punaro et al., (2014) detalharam em seu trabalho, os componentes do kefir, o que pode ser de grande valia para possíveis discussões científicas e até mesmo para melhor respaldo às intervenções clínicas. Logo, foram utilizados leite, leite em pó desnatado Molico (Nestlé®, São Paulo, Brasil), cultura láctica: kefir

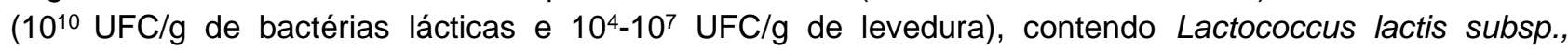
Leuconostoc sp., Streptococcus termophilus, Lactobacillus sp, levedura de kefir e microflora de grãos de kefir. Neste trabalho, a glicemia após a oitava semana reduziu significativamente $(p<0,001)$ nos ratos diabéticos tratados com a dose de 1,8 $\mathrm{mL} /$ dia de kefir (DMK) quando comparados aos animais diabéticos sem tratamento (DM) (325 \pm 32 vs $457 \pm 31 \mathrm{mg} / \mathrm{dL}$ ). Além do mais, durante o teste oral de tolerância a glicose (TOTG) observou-se que a glicemia no grupo DMK estava diminuída após 30, 60 e 120 minutos quando comparada ao DM, embora este resultado tenha sido significativo $(p<0,05)$ somente após $30 \mathrm{~min}(344 \pm 37 \mathrm{vs} 221 \pm 51$ $\mathrm{mg} / \mathrm{dL}$ ). Entre os achados do estudo, o tratamento com kefir em ratos diabéticos resultou não só em melhor controle glicêmico, mas também permitiu redução da poliúria, polidipsia e polifagia, único estudo exposto nesta revisão que avaliou a sintomatologia da doença apresentada pelos animais. E a melhora na sintomatologia pode ser de grande importância, se extrapolada aos humanos. 
No mesmo ano, Alsayadi et al. (2014) publicaram um estudo com o objetivo de avaliar as atividades antihiperglicêmicas e anti-hiperlipidêmicas do kefir de água por 35 dias. Após uma semana de intervenção, já foi possível observar redução da glicemia dos ratos diabéticos tratados com kefir na água. Sendo que ao final do experimento, a glicemia reduziu em $69 \%$ no grupo que recebeu $10 \%$ de kefir na água, $71 \%$ do grupo que recebeu dieta padrão com $20 \%$ de kefir e $63 \%$ do grupo que recebeu dieta padrão com $30 \%$ de kefir. Esses dados são interessantes pois, parece que acima de $20 \%$ de kefir na água, os benefícios se estabilizam. A redução da glicemia foi significativa $(p \leq 0,05)$ em todos os grupos tratados com kefir quando comparados aos ratos controle normais e diabéticos, porém não houve diferenças significativas entre os diferentes grupos kefir. Portanto, o consumo de kefir em água (10\% a 30\%) durante 5 semanas promoveu efeito benéfico na glicemia de ratos diabéticos.

Diferentemente dos estudos de Lee et al. (2011), Hadisaputro et al. (2012), Punaro et al., (2014) e Alsayadi et al. (2014) que pesquisaram os efeitos do kefir inoculado em água ou leite desnatado, Nurliyani et al. (2015a) avaliaram as propriedades do kefir preparado em combinação de leite de cabra e extrato de arroz preto e a sua influência na melhora das células $\beta$ pancreáticas em ratos diabéticos. Otles e Cagindi (2003) afirmaram que a matéria-prima de kefir é geralmente preparada a partir de leite de vaca, mas também pode ser preparada com outros leites como os de cabra e ovelha ou extratos vegetais, como os de coco, arroz ou soja. Após 28 dias de intervenção, o tratamento com kefir de extrato de arroz preto e leite de cabra nas doses de $2,0 \mathrm{ml}$ ou $4,0 \mathrm{ml}$ mostraram efeito significativo no número crescente de células de Langerhans e $\beta$, em comparação ao grupo tratado com a dose de $1,0 \mathrm{ml}$ de kefir ou aos ratos diabéticos não tratados. Por conseguinte, os autores sugerem ser necessário pelo menos a dose correspondente a $2,0 \mathrm{ml}$ de kefir inoculado em leite de cabra e extrato de arroz preto para causar efeitos positivos semelhantes à glibenclamida.

Em 2015, o estudo experimental de Muntafiah et al. (2015) verificaram o comportamento glicêmico de ratos induzidos ao diabetes. Para o tratamento neste estudo, foi inoculado $2 \%$ de sementes de de kefir, e a dosagem utilizada foi de $2 \mathrm{~mL} / 200 \mathrm{~g}$ de massa corporal. Após 4 semanas de intervenção, o grupo controle saudável aumentou a glicemia de jejum, mas ainda dentro de valores considerados normais $(<126 \mathrm{mg} / \mathrm{dL})$, ao passo que no grupo controle diabético a glicemia aumentou além da normalidade $>126 \mathrm{mg} / \mathrm{dL}(379,72 \pm 83,97$ $\mathrm{mg} / \mathrm{dL}$ ), enquanto os três grupos tratados com kefir demonstraram diminuição na glicemia de jejum, mas apenas o tratamento com a combinação de $50 \%$ kefir de leite de cabra e $50 \%$ kefir extrato de soja mostrou uma diminuição significativa na glicemia de jejum $(p=0,000)$. Para os autores, o mecanismo hipoglicemiante se da pela sinergia entre os componentes bioativos presentes, como exopolissacarídeos, peptídeos, antioxidantes, propriedades imunomoduladoras e bactérias probióticas, explicação também referida no estudos de Hadisaputro et al. (2012) e posteriormente no de Nurliyani et al. (2015a).

Em outro estudo, Nurliyani et al. (2015b) investigaram o efeito do kefir do leite de cabra, extrato de soja e a combinação de ambos no perfil lipídico, glicemia, atividade da glutationa peroxidase na melhora das células $\beta$ pancreáticas em ratos diabéticos por 35 dias. Em suma, os resultados evidenciados demonstraram redução da glicemia nos três grupos que foram administrados kefir. No entanto, a combinação de kefir de leite de cabra com extrato de soja e o kefir de extrato de soja proporcionaram reduções significativas na glicemia $(p<0,05)$ do que o kefir de leite de cabra. Este estudo também evidenciou que o número médio de ilhotas de Langerhans dos ratos diabéticos tratados com a combinação de kefir leite de cabra e extrato de soja foi maior do que no grupo kefir leite de cabra ou kefir extrato de soja, apesar de estatisticamente não serem significativos, no entanto, o grupo diabético tratado com leite de cabra e extrato de soja kefir apresentou maior $(p<0,05)$ número de ilhotas de Langerhans quando comparado aos diabéticos não tratados (controle positivo). Ademais, o número de células $\beta$ nos ratos diabéticos tratados com a combinação de leite de cabra e extrato de soja de kefir $(66,55 \pm 20,69)$ foi semelhante aos ratos saudáveis $(61,29 \pm 16,26)$. Resultado que corroborou aos achados reportados no outro estudo de Nurliyani et al. (2015a). Por fim, os autores deste estudo, a partir dos seus achados suportam que o kefir tem potencial para ser usado como intervenção coadjuvante na terapia do DM2. 
Sunarti et al. (2015) também investigaram os efeitos do leite de cabra com kefir, extrato de soja com kefir e combinação de ambos sobre os níveis de glicose por um período de 4 semanas em ratos diabéticos. Para o tratamento, foram inoculados grãos de kefir em até $2 \%$ nos leites de cabra, extrato de soja e na combinação de ambos ( $50 \%$ leite de cabra: $50 \%$ de extrato de soja). Após a indução do diabetes, os animais dos grupos 3,4 e 5 receberam diariamente $2 \mathrm{~mL} /$ dia de leite de cabra kefir, $2 \mathrm{~mL} /$ dia de extrato de soja kefir e $2 \mathrm{~mL} / \mathrm{dia}$ da combinação de ambos, enquanto os grupos 1 e 2 receberam água. Os achados demonstraram um decréscimo significativo na glicemia dos ratos diabéticos tratados com kefir de leite de cabra, sendo de 312,21 $\pm 87,12 \mathrm{mg} / \mathrm{dL}$ para $126,10 \pm 27,97 \mathrm{mg} / \mathrm{dL}(\mathrm{p}<0,05)$ e nos ratos diabéticos tratados com a combinação de kefir de leite de cabra e extrato de soja, sendo $338,41 \pm 41,75 \mathrm{mg} / \mathrm{dL}$ para $112,46 \pm 11,11 \mathrm{mg} / \mathrm{dL}(\mathrm{p}<0,05)$ após a intervenção, respectivamente. Enquanto, os animais diabéticos tratados com kefir de extrato de soja também apresentaram redução, mas não de maneira significativa, sendo de $391,24 \pm 146,58 \mathrm{mg} / \mathrm{dL}$ para 257,38 \pm $175,16 \mathrm{mg} / \mathrm{dL}$. Todavia, é importante considerar que, embora não tenha sido observada diferença significativa, clinicamente essa melhora é relevante para atenuação de outras desordens metabólicas. Em suma, os autores sugerem que a administração do kefir de leite de cabra ou a combinação do kefir do leite de cabra e extrato de soja podem reduzir significativamente a glicemia de ratos diabéticos, achados que se assemelham ao estudo anterior de Nurliyani et al., (2015b).

De fato, a partir dos estudos encontrados em modelo animal, a intervenção à base de kefir inoculado, seja em água, leite desnatado ou outros tipos de leite ou extratos vegetais, por períodos entre 30 à 56 dias mostrouse positiva na regulação glicêmica em diversas doses e diluições. Contudo, os mecanismos de ação são pouco elucidados, acreditando-se principalmente na hipótese inflamatória, tendo em vista que as citocinas pró-inflamatórias estimulam processos molecurares que diminuem a captação de glicose em tecidos dependentes de insulina e ainda, parecem prejudicar a secreção de insulina.

Todavia, os estudos em humanos são escassos, tendo em vista que apenas 2 estudos foram encontrados com possibilidade de análise crítica e extrapolação para verificar se os resultados suportam os achados apresentados e discutidos em modelo animal.

Judiono et al. (2014) conduziram um estudo clínico, de delineamento pré-teste e pós-teste com 108 indivíduos diabéticos do tipo 2 por 30 dias. Os pacientes foram divididos aleatoriamente em três grupos, grupo 1 (DM2, HbA1c $<7,0 \%$, com dieta padrão e suplementado com $200 \mathrm{~mL} /$ dia de kefir), grupo 2 (DM2, HbA1c $>7,0 \%$, com dieta padrão e suplementado com $200 \mathrm{~mL} /$ dia de kefir) e grupo 3 (DM2, com dieta padrão e sem administração de kefir, sendo o grupo controle). A glicemia em jejum e a glicose pós pradial diminuiram nos três grupos após intervenção, mas os grupos tratados com kefir demostraram diminuições na glicemia mais expressivas em relação ao grupo controle, havendo redução significativa na glicemia nos grupos 1 e 2 $(p=0,003)$. Em relação ao peptídeo $C$, o grupo 1 apresentou aumento médio de $0,68 \pm 0,18 \mathrm{pg} / \mathrm{mL}$, o grupo 2 de $0,07 \pm 0,12 \mathrm{pg} / \mathrm{mL}$, enquanto que o grupo não tratado reduziu em $0,02 \pm 0,06 \mathrm{pg} / \mathrm{mL}$. Ademais, os grupos tratados com kefir reduziram significamente a $\mathrm{HbA} 1 \mathrm{c}$ em comparação ao grupo controle após o período de intervenção. Houve reduções de $\mathrm{HbA} 1 \mathrm{c}$ de $0,13 \pm 0,15 \%$ e $0,09 \pm 0,24 \%$ nos grupos 1 e 2 , respectivamente, enquanto observou-se aumento de $0,001 \%$ nesse parâmetro no grupo controle. Segundo os autores, o kefir foi capaz de prevenir a glicotoxicidade e a lipotoxicidade, e ainda reduzir a ocorrência de hiperglicemia, estando em consonância aos achados do estudo anterior de Hadisaputro et al. (2012) realizado em animais.

O mecanismo subjacente proposto pelo autores é pela redução do estresse oxidativo, haja vista que o kefir diminuiu os níveis de peroxidação lipídica no sangue. Moléculas de peróxido podem afetar a produção de citocinas pró-inflamatórias, tais como IL-1, IL-6 e TNFa e, é visto que estas interleucinas prejudicam a via de sinalização da insulina, ativando uma reconhecida via pró-inflamatoria que promove a dissociação do IkB/NF-kB, levando ao dano e à apoptose das células $\beta$ pancreáticas. Outro mecanismo subjacente se dá provavelmente pelos exsopolissacarídeos biotativos (EPS) via c-AMP.Os EPS parecem ativar o hormônio glucagon, que é semelhante ao peptídeo 1 (GLP-1), peptídeo inibitório gástrico (GIP) e a enzima adenilato ciclase através da adenosina monofosfato cíclica (c-AMP), sensibilização de íons $\mathrm{Ca}^{2+}$ e ativação de proteína quinase A, por fim, aumentando assim a liberação de insulina das células $\beta$ pancreáticas. Em suma, acredita- 
se que, o aumento de c-AMP nas células pancreáticas parece contribuir para uma melhor secreção de insulina pelas células $\beta$ (JUDIONO et al. 2014).

Outro estudo realizado em indivíduos DM2 foi o de Ostadrahimi et al., 2015. Um estudo clínico, randomizado, duplo-cego, controlado por placebo, no qual recrutou 60 pacientes DM2 com idade entre 35 a 65 anos. Durante oito semanas, os pacientes do grupo intervenção receberam $600 \mathrm{~mL}$ de leite fermentado (kefir) contendo probióticos e o grupo placebo recebeu $600 \mathrm{~mL}$ de leite fermentado convencional (massa), ambos duas vezes ao dia (almoço e jantar). Foi constatado que no leite fermentado convencional (massa) haviam as cepas Streptococcus thermophiles e Lactobacillus bulgaricus, enquanto no leite fermentado kefir havia Streptococcus thermophiles enriquecido com Lactobacillus casei, Lactobacillus acidophilus e Bifidobacterium lactis. Entre os achados deste estudo, o grupo kefir reduziu significativamente a glicemia em jejum, de 161,63 $\pm 57,71$ para 139,22 $\pm 46,66 \mathrm{mg} / \mathrm{dL}$. O grupo kefir também apresentou redução significativa HbA1c após tratamento $(p=0,001)$, sendo o valor inicial de $7,61 \pm 1,22$ e final de $6,40 \pm 1,91 \%$, enquanto 0 grupo placebo não demonstrou diminuição deste parâmetro. Esta redução na hemoglobina glicada evidenciada por este estudo sugere um impacto clínico importante. Portanto houve maior controle glicêmico durante o período de intervenção do estudo, o que pode proporcionar redução das possíveis complicações micro e macrovasculares apresentadas por estes indivíduos a longo prazo. A partir de tais resultados, os autores acreditam que o leite fermentado kefir pode ser útil no manejo glicêmico e, assim, coadjuvante no tratamento das desordens glicêmicas. Porém, nenhum dos estudos citados aproundaram na relação com a MI. Assim, embora não tenha sido trago à tona essa relação pelos pesquisadores, hipotetizamos que, a regulação glicêmica observada na maioria dos estudos é oriunda de mudanças no equilíbrio microbiano do intestino e, por conseguinte, reduzindo a inflamação sistêmica, melhorando a captação de glicose em tecidos periféricos, em especial, no músculo esquelético.

\section{CONCLUSÃO}

As pesquisas experimentais, apesar da grande heterogeneidade dos protocolos, demonstraram efeitos positivos em diversos marcadores bioquímicos associados a desordens glicêmicas após intervenções baseadas no kefir. No entanto, foram encontrados poucos estudos realizados em humanos diabéticos e com limitações metodológicas, porém os resultados encontrados são promissores. Assim, parece que o kefir apresenta potencial para ser utilizado como coadjuvante no manejo das desordens glicêmicas a curto, médio e longo prazo, entretanto, mais estudos clínicos, randomizados e controlados são necessários para confirmação, principalmente no que diz respeito às análises relacionadas à microbiota, para que se possa compreender de forma mais completa a tríade composta por: kefir, microbiota intestinal e regulação glicêmica.

\section{REFERÊNCIAS}

1. ALSAYADI M, AL JAWFI Y, BELARBI M et al. Evaluation of anti-Hyperglycemic and anti-hyperlipidemic activities of water kefir as probiotic on Streptozotocin-induced diabetic Wistar rats. Journal of Diabetes Mellitus, 2014; 4.02: 85.

2. AMERICAN DIABETES ASSOCIATION. 2. Classification and diagnosis of diabetes: standards of medical care in diabetes2018. Diabetes Care, 2018, $41 \mathrm{p}$.

3. BOURRIE BCT, WILLING BP, COTTER PD. The microbiota and health promoting characteristics of the fermented beverage kefir. Frontiers in Microbiology, 2016; 7: 647.

4. CHATTERJEE S, KHUNTI K, DAVIES M. Type 2 diabetes. The Lancet, 2017; 389.10085: 2239-2251.

5. DE FILIPPIS F, PELLEGRINI N, VANNINI $L$ et al. High-level adherence to a Mediterranean diet beneficially impacts the gut microbiota and associated metabolome. Gut, 2016; 65(11):1812-1821.

6. EJTAHED HS, SOROUSH AR, ANGOORANI P et al. Gut microbiota as a target in the pathogenesis of metabolic disorders: a new approach to novel therapeutic agents. Hormone and Metabolic Research, 2016; 48.06: 349-358.

7. FOOD AND AGRICULTURAL ORGANIZATION OF UNITED NATIONS/WORLD HEALTH ORGANIZATION. Joint expert consultation on evalution health and nutritional properties of probiotics in food including powder milk with live lactic acid bacteria. Córdoba, Argentina; October 1-4, 2001. 
8. FORSLUND K, HILDEBRAND F, NIELSEN T et al. Disentangling type 2 diabetes and metformin treatment signatures in the human gut microbiota. Nature, 2015, 528.7581: 262.

9. HADISAPUTRO S, DJOKOMOELJANTO RRJ, SOESATYO MH et al. The effects of oral plain kefir supplementation on proinflammatory cytokine properties of the hyperglycemia Wistar rats induced by streptozotocin. Acta Med Indones, 2012; 44.100: e4.

10. HE J, ZHANG F, HAN Y. Effect of probiotics on lipid profiles and blood pressure in patients with type 2 diabetes: A meta-analysis of RCTs. Medicine (Baltimore), 2017; 96(51): e9166.

11. HSU YJ, HUANG WC, LIN JS et al. Kefir Supplementation Modifies Gut Microbiota Composition, Reduces Physical Fatigue, and Improves Exercise Performance in Mice. Nutrients, 2018; 4;10(7).

12. JOHN SM, DEESEENTHUM S. Properties and benefits of kefir-A review. Songklanakarin Journal of Science \& Technology, $2015 ; 37.3$.

13. JUDIONO J, HADISAPUTRO S, INDRANILA KS et al. Effects of clear kefir on biomolecular aspects of glycemic status of type 2 diabetes mellitus (T2DM) patients in Bandung, West Java [study on human blood glucose, c peptide and insulin]. Functional Foods in Health and Disease, 2014; 4.8: 340-348.

14. KADOOKA Y, SATO M, OGAWA A et al. Effect of Lactobacillus gasseri SBT2055 in fermented milk on abdominal adiposity in adults in a randomised controlled trial. British Journal of Nutrition, 2013; 110(9), 1696-1703.

15. KHALESI, Saman, et al. A review of probiotic supplementation in healthy adults: helpful or hype?. European journal of clinical nutrition, 2018, 1.

16. LEE J, SONG KY, CHON JW. Effects of oral administering kefir on blood glucose levels in diabetic mice. The Korean Journal of Food and Nutrition, 2011; 24.1: 79-84.

17. MAGALHÃES KT, PEREIRA GVDM, CAMPOS CR et al. Brazilian kefir: structure, microbial communities and chemical composition. Brazilian Journal of Microbiology, 2011; 42.2: 693-702.

18. MITTAL K, MANI RJ, KATARE, DP. Type 3 diabetes: cross talk between differentially regulated proteins of type 2 diabetes mellitus and Alzheimer's disease. Scientific Reports, 2016; 6: 25589.

19. MUNTAFIAH A, et al. POTENSI ANTIHIPERGLIKEMIA KEFIR BERBASIS SUSU KAMBING DAN KEDELAI PADA TIKUS MODEL DM TIPE 2. Mandala of Health A Scientific Journal, 2015; 8 (3): 612-621.

20. NURLIYANI, HARMAYANI E, SUNARTI. Antidiabetic potential of kefir combination from goat milk and soy milk in rats induced with streptozotocin-nicotinamide. Korean Journal for Food Science of Animal Resources, 2015; 35.6: 847.

21. NURLIYANI, SADEWA AH, SUNARTI. Kefir Properties Prepared with Goat Milk and Black Rice (Oryza sativa L.) Extract and its Influence on the Improvement of Pancreatic $\beta$-Cells in Diabetic Rats. Emirates Journal of Food and Agriculture, 2015; 27.10: 727-735.

22. OSTADRAHIMI A, TAGHIZADEH A, MOBASSERI M et al. Effect of probiotic fermented milk (kefir) on glycemic control and lipid profile in type 2 diabetic patients: a randomized double-blind placebo-controlled clinical trial. Iranian Journal of Public Health, 2015; 44.2: 228.

23. OTLES S, CAGINDI O. Kefir: a probiotic dairy-composition, nutritional and therapeutic aspects. Pakistan Journal of Nutrition, 2003; 2.2: 54-59.

24. PEREIRA TMC, PIMENTA FS, PORTO ML et al. Coadjuvants in the Diabetic Complications: Nutraceuticals and Drugs with Pleiotropic Effects. International Journal of Molecular Sciences, 2016; 17(8):1273.

25. PUNARO GR, MACIEL FR, RODRIGUES AM et al. Kefir administration reduced progression of renal injury in STZ-diabetic rats by lowering oxidative stress. Nitric Oxide, 2014; 37: 53-60.

26. SOCIEDADE BRASILEIRA DE DIABETES. Diretrizes da sociedade brasileira de diabetes 2017-2018, 2017, 383 p.

27. SUNARTI, NURLIYANI, TYAS ASA et al. The Influence of Goat Milk and Soybean Milk Kefir On IL-6 and CRP Levels in Diabetic Rats. Romanian Journal of Diabetes Nutrition and Metabolic Diseases, 2015; 22.3: 261-267.

28. TAMANG JP, WATANABE K, HOLZAPFEL WH. Review: Diversity of Microorganisms in Global Fermented Foods and Beverages. Frontiers in Microbiology, 2016; 7, 377.

29. TORRES-FUENTES C et al. The microbiota-gut-brain axis in obesity. The lancet Gastroenterology \& Hepatology, 2017; 2(10), 747-756.

30. WALSH AM, CRISPIE F, KILCAWLEY $\mathrm{K}$ et al. Microbial succession and flavor production in the fermented dairy beverage kefir. Msystems, 2016; 1.5: e00052-16.

31. ZHENG Y, LEY SH, HU FB. Global aetiology and epidemiology of type 2 diabetes mellitus and its complications. Nature Reviews Endocrinology, 2018; 14(2), 88-98. 